\title{
The Role of Sympathetic System as a Therapeutic Option in the Ischemia/Reperfusion Injury
}

\author{
Bertha F. Polegato ${ }^{1}$ and Leonardo A. M. Zornoff' ${ }^{10}$ \\ Universidade Estadual Paulista (Unesp) - Faculdade de Medicina de Botucatu - Departamento de Clínica Médica, ${ }^{1}$ Botucatu, SP - Brazil \\ Short Editorial related to the article: Acute Physical Stress Preconditions the Heart Against Ischemia/Reperfusion Injury Through Activation of \\ Sympathetic Nervous System
}

Coronary artery disease is the leading cause of mortality and the most resource-consuming health pathology in industrialized countries. ${ }^{1}$ In the United States, it is believed that more than 12 million individuals have ischemic heart disease. ${ }^{2}$

It is estimated that approximately 1 million cases of acute coronary syndrome occur annually in the United States, demonstrating that this syndrome occurs in epidemic proportions. ${ }^{2}$ Few pathologies have evolved as radically as AMI, with a marked reduction in mortality as a result of changes in treatment over the past 30 years, particularly cardiac reperfusion. ${ }^{1,3}$

In fact, with the introduction of myocardial reperfusion, 30 -day mortality has been reduced from about $14 \%$ to about $3 \%$ in several clinical trials. ${ }^{3}$ In addition to this benefit, early and sustained reperfusion results in lower cardiac morbidity, lower incidence of ventricular fibrillation and tachycardia, conduction disorders and less development of congestive heart failure. ${ }^{1}$

Despite the unequivocal benefit, an undesirable event of this strategy is the phenomenon of reperfusion injury.

\section{Keywords}

Stress, Mechanical; Simpathetic Nervous System; Hypothalamo-Hpophyseal System; Ischemia; Sympathectomy.

\section{Mailing Address: Leonardo A. M. Zornoff •}

Departamento de Clínica Médica - Faculdade de Medicina de Botucatu Universidade Estadual Paulista (Unesp). Postal Code 18618-970, Botucatu, $\mathrm{SP}-$ Brazil

E-mail: Izornoff@fmb.unesp.br

DOI: $10.5935 / a b c .20190183$
This phenomenon is defined as the injury that occurs as a direct result of coronary blood flow restoration. This phenomenon may have important clinical implications, because it may be responsible for $30-50 \%$ of the final infarct size. ${ }^{4}$ Thus, several strategies have been studied with the objective of attenuating the ischemia/reperfusion (I/R) injury phenomenon.

In this issue of the Arquivos Brasileiros de Cardiologia, Imani et al. ${ }^{5}$ assessed the cardioprotective effects of acute physical stress against I/R injury, through the activation of the sympathetic nervous system. They used the isolated heart preparation, with the Langendorff apparatus. The hearts were subjected to 30 minutes of ischemia, followed by 120 minutes of reperfusion. Physical stress prior to the $\mathrm{I} / \mathrm{R}$ improved left ventricular developed pressure and reduced infarct size when compared with the I/R alone. ${ }^{5}$ In addition, chemical sympathectomy before physical stress eliminated the protective effect of physical stress on I/R-induced cardiac damages. The authors concluded that the presence of the sympathetic nervous system is necessary for the beneficial effects of acute physical stress on I/R injury. ${ }^{5}$

It is important to emphasize that knowledge about the pathophysiological mechanisms involved in $\mathrm{I} / \mathrm{R}$ injury is critical, as this allows the creation of therapeutic strategies to attenuate or prevent cardiac damage. On the other hand, we must consider that cardioprotection strategies in $\mathrm{I} / \mathrm{R}$ models are the main model used to exemplify the difficulties of translational medicine, since positive results from experimental studies are obfuscated by the fact that to date, cardioprotection strategies in clinical studies have shown negative results. ${ }^{6}$

Therefore, although provocative, the role of the sympathetic system as a therapeutic option in the I/R injury remains to be confirmed in future studies.

\section{References}

1. Ibanez B, James S, Agewall S, Antunes MJ, Bucciarelli-Ducci C, Bueno H, et al. 2017 ESC Guidelines for the management of acute myocardial infarction in patients presenting with ST-segment elevation. Eur HeartJ. 2018;39(2):119-77.

2. Hedayati T, Yadav N, Khanagavi J. Non-ST-segment acute coronary syndromes. Cardiol Clin. 2018;36(1):37-52.

3. Puymirat E, Simon T, Cayla G, Cottin Y, Elbaz M, Coste P, et al. Acute myocardial infarction: changes in patient characteristics, management, and 6-month outcomes over a period of 20 years in the FAST-MI Program (French Registry of Acute ST-Elevation or Non-ST-Elevation Myocardial Infarction) 1995 to 2015. Circulation. 2017;136(20):1908-19.

4. Yellon DM, Hausenloy DJ. Myocardial reperfusion injury. N Engl J Med. 2007;357(11):1121-35.

5. Imani A, Parsa H, Chookalaei LG, Rakhshan K, Golnazari M, Faghihi M. Acute Physical Stress Preconditions the Heart Against Ischemia/Reperfusion Injury Through Activation of Sympathetic Nervous System. Arq Bras Cardiol. 2019; 113(3):401-408.

6. Garcia LR, Polegato BF, Zornoff LAM. Challenges of translational science. Arq Bras Cardiol 2017;108(5):388-9. 